Proceedings of the 2001 IEEE

International Conference on Robotics \& Automation

Seoul, Korea - May 21-26, 2001

\title{
A Controllability Test and Motion Planning Primitives for Overconstrained Vehicles
}

\author{
T. D. Murphey, J. W. Burdick \\ Engineering and Applied Science, California Institute of Technology \\ Mail Code 104-44, Pasadena, CA 91125 USA \\ \{murphey,jwb\}@ robotics.caltech.edu
}

Keywords: Overconstrained wheeled vehicles, switched systems, controllability, motion planning.

\begin{abstract}
Conventional nonholonomic motion planning and control theories do not directly apply to "overconstrained vehicles," such as the Sojourner vehicle of the Mars Pathfinder mission. This paper discusses some basic issues of motion planning and control for this pctentially important class of mobile robots. A power dissipation approach is used to model the governing equations of overconstrained vehicles that move quasi-statically. These equations are shown to be switched hybrid systems. Notions from standard geometric control, such as the Lie bracket, are extended to these switched systems. We then develop a controllability test for such systems. We explore motion planning primitives in the context of simplified examples.
\end{abstract}

\section{Introduction and Motivation}

Most mobile robots use wheels since they provide one of the simplest means for mobility. Since wheels impose nonholonomic constraints on a vehicle's motion, the subject of control and motion planning for nonholonomic wheeled vehicles has been widely pursued $[1,2]$. In order to operate in moderately rough terrains without resorting to the inherent complexity of legged mechanisms, "overconstrained" wheeled vehicle designs have been proposed. The most famous example is the Sojourner robot deployed during the Pathfinder mission to Mars. Fig. 1 shows the "Rocky 7," a prototype for future Mars rover vehicles whose suspension and wheel kinematics are essentially identical to the Sojourner. The Rocky 7 employs six wheels, with both front wheels independently steered and all six wheels independently driven, making it an eight input system. The rear wheels on each side are coupled through a "bogey" linkage mechanism that helps the vehicle negotiate obstacles that are up to 1.5 times the wheels' diameter. Below we shall show that standard nonholonomic motion planning and control theories can not be applied to this vehicle.

To motivate the issues that are considered in this paper, consider a highly simplified model of the Rocky 7 vehicle (Fig. 2(b)). In this stripped down model, hereafter referred

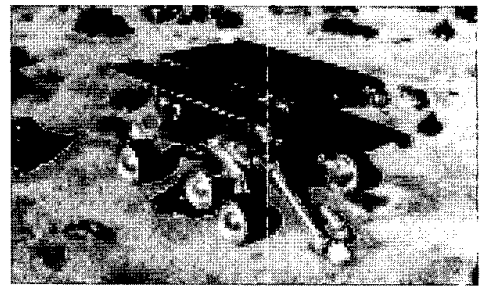

Figure 1: Photo of Rocky 7 Mars Rover Prototype

to as the Simplified Rocky 7 (or SR7), the vehicle operates on flat terrain. To realize the model of Fig. 2, each pair of Rocky 7 wheels is conceptually "collapsed" into a single wheel, as is done in conventional models of the classical kinematic car (Fig. 2(a)). We further assume that only the front wheel is actuated. While highly simplified, this model captures many of the essential features and challenges of overconstrained wheeled vehicles. That the Rocky 7 operates in non-planar terrain and has additional wheel actuation will pose further complexities.

The motion of every planar body can be characterized at each instant by its Instantaneous Center of Rotation (ICR). In the classical kinematic car model (Fig. 2(a)), the assumption that the wheels do not slip defines an instantaneous center of rotation at the intersection of the lines that are collinear with the two wheel axes. Note that because of the overconstraint due to the presence of an additional wheel, kinematic issues alone can not be used to determine the ICR of the SR7 vehicle in Fig. 2(b). Standard nonholonomic motion planning and control (NMPC) techniques implicitly assume that the ICR is uniquely defined by non-slip wheel constraints that can described by purely kinematic relationships. Furthermore, the overconstraint implies that unless the vehicle is stopped or moving straight ahead, one or more wheels are always slipping-i.e, the no-slip assumption that is at the basis of NMPC techniques is violated.

One might argue that the "extra" wheels in the systems of Fig.s 1 and 2(b) can be practically ignored. After all, 18wheeled trucks have similarly overconstrained geometries. However, we seek dextrous maneuvering of such robots far beyond that which is required for 18-wheelers. For example, future rover mission scenarios call for a manipulator arm with only 2 or 3 degrees of freedom (e.g., Fig. 1) to 

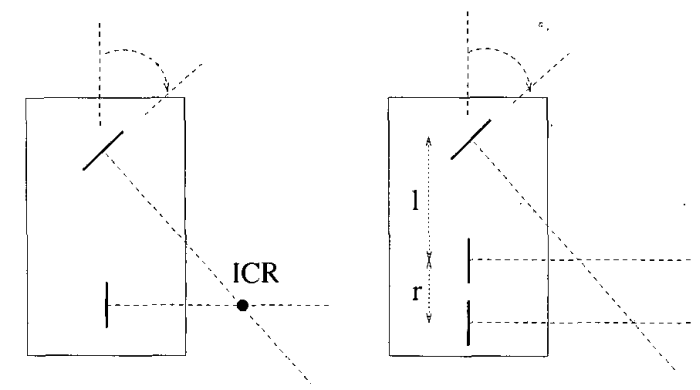

Figure 2: (a) kinematic car; (b) simplified Rocky 7.

collect rock samples and position sensors. Arbitrary displacements of the arm's end-effector will require the vehicle to make local sideways motions so as to compensate for the arm's kinematic deficiency.

As represented by Mars exploration opportunities, overconstrained vehicles are a potentially important class of robots. We seek to develop theories and algorithms that parallel those for nonholonomic mobile robots. Currently, no such systematic control and motion planning theory exists. This paper makes some first steps in this direction. We first consider how to model such systems for the purpose of motion planning. A power dissipation model suggested by Alexander and Maddocks [3] is used to develop the governing equations. We formalize the techniques suggested in their paper and show that the resulting equations have the structure of a switched hybrid system. We next show how to extend notions such as the Lie bracket to the switched case. Based on these extensions, we develop a small-time local controllability test for such systems. This test answers the practical question: "can the overconstrained vehicle locally move in any direction?" Controllability tests for smooth nonholonomic systems are well known [4], and analogous tests have been developed for some other types of hybrid systems [5]. To our knowledge, this is the first controllability test for such systems. Finally, in the context of the model of Fig. 2(b), we consider motion planning primitives for overconstrained mobile robots.

\section{Mathematical Preliminaries}

We assume the reader is familiar with the basic formalism of differential geometry and nonlinear control theory. We remind the reader of the following definitions and classical theorems so that it may be clear where we are starting from. Let $Q$ be an $n$-dimensional configuration space, $T Q$ the tangent bundle, and $\mathcal{U}$ the $m$-dimensional space of inputs. We denote the function space of the coefficient of friction by $\Xi$, the function space of wheel normal forces by $\mathcal{N}$, and the function space of switching signals by $\Sigma$.

The mechanics of classical nonholonomic systems can be put into the form of a drift free affine system:

$$
\dot{q}=g_{1}(q) u_{1}+g_{2}(q) u_{2}+\cdots+g_{m}(q) u_{m}
$$

where $q \in \mathbb{R}^{n}$ is the system's state space, $\left(u_{1}, \ldots, u_{m}\right)$ are the controls, and $g_{1}, \ldots, g_{m}$ are termed the control vectors fields. Local controllability is roughly the ability to, in an open neighborhood of a starting point, find inputs that steer the system to any configuration in that neighborhood. To achieve this for linearly uncontrollable systems, the Lie Bracket $[f, g]$ between two vector fields $f$ and $g$,

$$
[f, g](q)=\frac{\partial g}{\partial q} f(q)-\frac{\partial f}{\partial q} g(q)
$$

must be used. We also define the involutive closure $\bar{\Delta}$ of a distribution $\Delta$ of vector fields as the closure of $\Delta$ under the Lie bracketing operation.

Theorem 1 (Chow) The control system (1) is small time locally controllable at $q \in \mathbb{R}^{n}$ if $\bar{\Delta}_{q}=T_{q} \mathbb{R}^{n}$.

The condition in Chow's theorem is equivalent to the reachable set (denoted $\mathcal{R}^{V}$ in the following) having nonempty interior.

Definition 1 Given an open set $V \subseteq \mathbb{R}^{n}$, let $\mathcal{R}^{V}\left(q_{0}, \tau\right)$ be the set of states $\dot{q}$ such that $\exists u:[0, T] \rightarrow \mathcal{U}$ steers the system from $q(0)=q_{0}$ to $q(T)=q_{f}$ and satisfies $q(t) \in V$ for $0 \leq t \leq T$. Moreover, define:

$$
\mathcal{R}^{V}\left(q_{0}, \leq \dot{T}\right)=\bigcup_{0<\tau \leq T} \mathcal{R}^{V}\left(q_{0}, \tau\right)
$$

As modeled using the power dissipation method of Section 3 , the motions of overconstrained wheeled vehicles are governed by switched systems, a class of hybrid dynamical systems. One of the purposes of this paper is to extend Chow's theorem to switched systems.

Definition 2 A system is a switched driftless affine system (SDA) if it can be expressed in the form

$$
\dot{q}=f_{1} u_{1}+f_{2} u_{2}+\cdots+f_{n} u_{n}
$$

where for any $x$ and $t, f_{i} \in\left\{g_{\alpha_{i}} \mid \alpha_{i} \in I_{i}\right\}$, with $I_{i}$ an index set and $f_{i}$ measurable in $(q, t)$ and $g_{i}$ analytic in $(q, t)$ for all $i$.

Since the composition of measurable functions is again measurable, one can equivalently consider $\alpha_{i}$ to be a function of $(q, t)$ and say that $f_{i}=g_{\alpha_{i}}$.

Definition 3 The multiplicity of a switched vector is defined as the number of elements in $I_{i}$, and the multiplicity of a switched driftless affine system is defined to be the maximum number of elements of the $I_{i}$ for all $i$.

For clarity and practicality we focus on switched driftless affine systems of multiplicity two. However, most of our results hold for higher multiplicity SDA systems.

\section{Models for Motion Planning}

We seek models for overconstrained systems that capture the essential physics of the problem, and that are tractable and amenable to control and motion planning analysis. This section briefly summarizes results from [6], which considers 
both the Lagrangian approach and in more detail a powerdissipation approach that is appropriate for quasi-statically moving vehicles, such as the Rocky 7 in Fig. 1. We assume that wheel contact with the ground is governed by the Coulomb friction law.

First, we consider the Lagrangian case, and explain why we choose not to use it as our modeling methodology. Constrained mechanical systems can be modeled using conventional Lagrangian mechanics via the use of Lagrange multipliers. Let $L(q, \dot{q})$ denote the Lagrangian (kinetic minus potential energy) of the vehicle system. If the $i^{t h}$ wheel is not slipping, then this constraint on the vehicle's motion can be expressed as $\omega_{i}(q) \dot{q}=0$. If the $i^{t h}$ wheel is slipping, then the Coulomb law governs the reaction force at that wheel: $F_{i}^{R}=-\frac{v_{i}}{\left\|v_{i}\right\|} \mu_{i} N_{i}$, where $\mu_{i}, N_{i}$, and $v_{i}$ are respectively the Coulomb friction cciefficient, normal force to the ground, and slipping velocity of the wheel at the $i^{t h}$ contact. Hence, the system's equations of motion are described by:

$$
\frac{d}{d t}\left(\frac{\partial L}{\partial \dot{q}}\right)-\frac{\partial L}{\partial q}+\sum_{i \in \mathcal{S}} F_{i}^{R}+\sum_{j \notin \mathcal{S}} \lambda_{j} \omega_{j}^{T}(q)=T
$$

where $\mathcal{S}$ is the slipping set, the $\left\{\lambda_{j}\right\}$ are undetermined Lagrange multipliers, and $T$ are generalized applied forces. That is, $k \in \mathcal{S}$ if the $k^{\text {th }}$ wheel contact is slipping. If the $k^{t h}$ wheel is not slipping, $\lambda_{k}$ corresponds to the reaction force necessary to maintain that no-slip constraint. There are two practical problems with the Lagrangian approach. First, one must solve for the Lagrange multipliers-a tedious task that often leads to complex equations. Second, an additional (and often complicated) analysis is necessary to determine which wheels are slipping at any given instant. After considering the power dissipation approach, we will return to the Lagrangian case and compare the two methods.

Many overconstrained vehicles, such as the Rocky 7 whose speed is measured in 10's of centimeters per second, move slowly enough that their motion can be considered quasi-static. Hence, it makes sense to develop a modeling approach that is suited to this realistic situation. Recall that at least one wheel always slips during overconstrained vehicle motion, thereby dissipating energy. The power dissipation method assumes that the system's motion at any given instant is the one that instantaneously minimizes power dissipation due to wheel slippage. This method is adapted from Alexander and Maddocks [3]. The power dissipation function measures the vehicle's total dissipation due to wheel slippage.

Definition 4 The Dissipation or Friction Functional for an $n$-wheeled vehicle is defined to be

$$
\begin{gathered}
\mathcal{D}=\sum_{i=1}^{n} \alpha_{i}|\omega(q) \dot{q}| \\
\text { where } \alpha_{i}=\mu_{i} N_{i} \text {, with } N_{i} \text { the } i^{t h} \text { normal force. }
\end{gathered}
$$

\subsection{The Power Dissipation Modeling Approach Leads to Switched Hybrid Systems.}

Ideally the dissipation function defined in Definition 4 would always have a unique minimum. Unfortunately, a unique minimum can not be expected.

Alexander and Maddocks [3] show that the dissipation model is convex, so local minima are global minima, should they exist. They also show that if they exist, they exist at a point of nondifferentiability of $\mathcal{D}$. This is not, however, sufficient for the uniqueness of such a kinematic state. Should two kinematic states, $\dot{q}_{1}$ and $\dot{q}_{2}$, minimize the dissipation functional $\mathcal{D}$ from Def. 4 , then additionally the convex hull $\operatorname{co}\left\{\dot{q}_{1}, \dot{q}_{2}\right\}$ will also do so. However, in [6] we showed that this only happens on a set of measure zero, and that therefore $\mathcal{D}$ has a unique minimum almost always. The minimization of $\mathcal{D}$ therefore generically leads to a switched system of the form in Definition 2 [6]. Moreover, when the vehicle is not overconstrained, the dissipation method leads to the classical nonholonomic kinematic equations.

To compare the PDM method to conventional Lagrangian analysis, consider a bicycle with torque inputs on both the front wheel $A$ and the back wheel $B$. An analysis based on Lagrangian mechanics would suggest that there are up to four possible contact states, corresponding to a) A slipping, b) B slipping, c) neither slipping, d) both slipping. On the other hand, the PDM analysis, using velocities as the wheel inputs instead of torques, suggests that there are only two different contact states corresponding to either A or B slipping. Possibilities c) and d) both imply that the inertial terms dominate the system's dynamics, thereby violating the quasi-static assumption. Case d) implies that the bicycle is skidding out of control, which violates our quasistatic assumption. The conditions corresponding to state c) are unlikely to be found in an actual system, as this implies that both contacts must be driven at exactly compatible speeds, or the normal forces are so high that they dominate the contact speeds instead of the contact speeds dominating them. In the case of c) where the speeds are exactly the same speed, the dimension of the subspace spanned by the constraints drops in any case, implying that constraint is essentially duplicated. Therefore the power dissipation will give results satisfying this constraint even if it is practically unlikely. This leaves the second two states, which are the same as what we found in the power dissipation model. This is an indication of how the quasi-static assumption helps to simplify our problem, while yielding similar insights to Lagrangian analysis.

\subsection{Model for the simplified Rocky 7}

Using the dissipation approach, we first show using [3] that the minimum of $\mathcal{D}$ must occur when either the middle or back wheel slips. If the configuration of the simplified Rocky 7 is $q=[x, y, \theta]^{T}$ and the controls $u_{1}$ and $u_{2}$ are associated with the drive velocity and steering velocity respectively, we find that the equations that govern the motion 
of the simplified Rocky 7 are:

$$
\begin{aligned}
& \dot{q}=g_{\sigma}(q) u_{1}+g_{3}(q) u_{2} \quad \sigma:(q, t) \rightarrow\{1,2\} \\
& g_{1}=\left[\begin{array}{c}
\cos (\psi) \cos (\theta) \\
\cos (\psi) \sin (\theta) \\
\frac{1}{l} \sin (\psi) \\
0
\end{array}\right] g_{2}=\left[\begin{array}{c}
\frac{l \cos (\psi-\theta)+(l-2 r) \cos (\psi+\theta)}{2(l-r)} \\
\frac{l \cos (\psi) \sin (\theta)-r \sin (\psi+\theta)}{l-r} \\
\frac{1}{l} \sin (\psi) \\
0
\end{array}\right] \\
& g_{3}=\left[\begin{array}{llll}
0 & 0 & 0 & 1
\end{array}\right]^{T}
\end{aligned}
$$

Our system is an SDA system as in Definition 2, with $\sigma$ : $(x, t) \rightarrow\{1,2\}$ (i.e., only two possible dynamic states). The function which determines the current state is:

$$
\Psi(g)=\left(\frac{\alpha_{1}}{\alpha_{2}}\right)^{2}\left(\frac{l-r}{r}\right)^{2}-1 .
$$

When $\Psi(g)>0, \sigma=1$; when $\Psi(g)<0, \sigma=2$. This formula implies that unless the $\mu(x)$ is know for all $x$, the current vehicle state is unknown. This observation suggests the practical use of wheel slip sensors.

\section{Controllability for Switching Systems}

We now address the issue of controllability, i.e, can the overconstrained vehicle locally move in any direction? There are two important cases to distinguish in the controllability of overconstrained vehicles: when the switching is controlled and when the switching is not under the vehicle's control. For the case of controlled switching, which requires a modest extension of controllability theory, see [6] for more details. Note that controlled switching of linear systems has been considered in [7]. The other case requires new definitions.

\subsection{Controlled Switching}

Let $\bar{\Delta}_{\sigma_{i}}$ denote the involutive closure of the control vector field distribution associated with switching state $\sigma_{i}$. When $\bar{\Delta}_{\sigma_{i}}$ is full rank, controllability is immediately realized, as one can (with the assumption of complete control over the switching process) switch to the controllable state $\sigma_{i}$. Conventional results for smooth systems then apply to this state. However, if none of the $\bar{\Delta}_{\sigma_{i}}$ are full rank, then controllability may still exist, but is not obvious. To motivate this situation, consider the example in Fig 3. This fixed

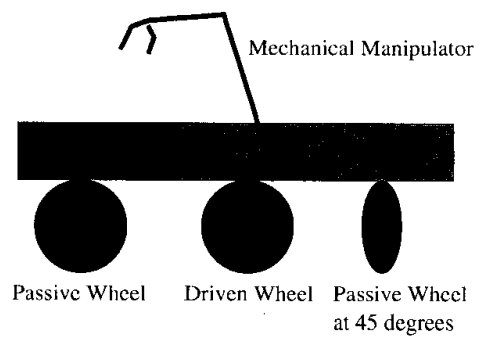

Figure 3: The Fixed Wheel Kinematic Car

wheel kinematic car (FWKC) has three wheels, of which only the middle is driven. None of the wheels are steerable: the back one remains straight, and the front remains at a constant angle of $\pi / 4$. We include the mechanical arm above the body as an example of a mechanism that can control switching. As the arm moves forward and backward, it can shift the center of mass sufficiently to switch the vehicle into a new dynamic state-i.e., the arm position determines which wheel is slipping. Each dynamic state by itself is uncontrollable, though we shall see that this system is indeed controllable. While this example generally has no practical value, it is illustrative of the idea, and may possibly represent the vehicle in a singular configuration of its suspension or a state of steering actuator failure. Based on the power dissipation approach, the governing dynamics of this vehicle are

$$
\begin{gathered}
\dot{q}=g_{\sigma}(q) u_{1} \quad \sigma:(q, t) \rightarrow\{1,2\} \\
g_{1}=\left[\begin{array}{c}
\frac{1}{\sqrt{2}} \cos (\theta) \\
\frac{1}{\sqrt{2}} \sin (\theta) \\
1
\end{array}\right], \quad g_{2}=\left[\begin{array}{c}
\cos (\theta) \\
\sin (\theta) \\
0
\end{array}\right] .
\end{gathered}
$$

Recall that the classical Lie bracket between two differentiable vector fields, which does not have any meaning in this switched system context, is equivalent to:

$$
[f, g](q)=\lim _{\varepsilon \rightarrow 0} \frac{1}{\varepsilon^{2}}\left(\Phi_{\varepsilon}^{-f} \circ \Phi_{\varepsilon}^{-g} \circ \Phi_{\varepsilon}^{f} \circ \Phi_{\varepsilon}^{g}(q)-q\right)
$$

where $\Phi_{\varepsilon}^{f}$ represents the flow along $f$ for time $\varepsilon$. The total flow can be seen schematically in Fig. 4.

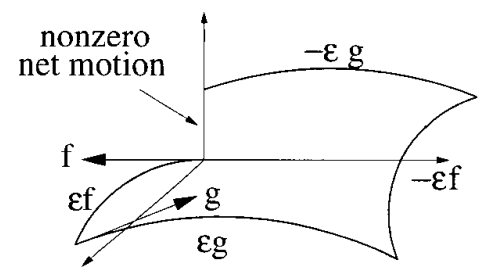

Figure 4: Flows associated with a Lie bracket motion.

The interpretation of the Lie bracket as a flow makes the following extension to the switched case almost trivial. Rather than forming the Lie Bracket between two separate smooth control input vector fields, we form a Lie Bracket of a control input vector field $f_{i} u_{i}$ where $f_{i} \in\left\{g_{i} \mid i \in\{1,2\}\right\}$. We do this by setting $u=1$ for $0 \leq t<2 \varepsilon$ and $u=-1$ otherwise, while $i=1$ for $0 \leq t<\varepsilon$ and $3 \varepsilon \leq t \leq 4 \varepsilon, i=2$ otherwise. This produces the flow seen in Figure 4 . This simple controlled switched Lie bracket(CSLB) can therefore be used to control the mechanism. This leads us to the following corollary of Chow's Theorem.

Corollary 1 Consider an SDA system of the form Definition 2, where the switching can be controlled directly. Let $\Delta_{\sigma_{i}}$ denote the distribution of the control vector fields associated with state $\sigma_{i}$. Let $\bar{\Delta}_{H, \sigma}$ denote the involutive closure of $\cup_{i} \Delta \sigma_{i}$. The system is locally controllable if $\bar{\Delta}_{H, \sigma}=T_{q} \mathbb{R}^{n}$ for all $q$. 
The proof of this corollary is sirnilar to a standard proof of Chow's Theorem (see Ref. [4] for example) with the modification that the flows are produced by a switching vector field. For this reason we omit details of the proof. Applying this result to the FWKC, we find the Lie bracket of the two vector fields to be $[-\sin \theta, \cos \theta, 0]^{T}$, which leads to a full span of the three-dimensional vector space. Therefore the FWKC is controllable. Simulations in Section 5 bear out this result.

\subsection{Uncontrolled Switching;}

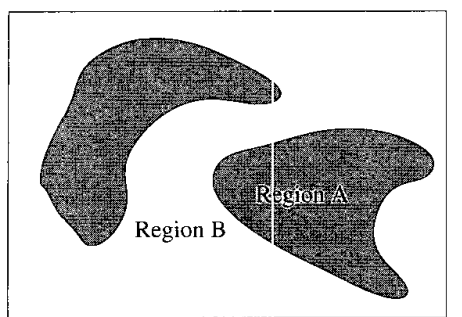

Figure 5: Cellular Separation of Kinematic States

To introduce uncontrolled switching, consider Fig. 5. Here we have two different regions, A and B, corresponding to the different kinematic states. The differences in these regions may arise from changes in the coefficient of friction or distribution of the vehicle's mass. As the mechanism goes from one region to the other its gcverning equations switch. The difficulty lies in the fact that the geometries of these regions may be completely unkriown, and moreover may be local in nature- i.e. Fig. 5 may correspond to an arbitrarily small neighborhood of the operating point. Our goal, then, is to have a theory which incorporates the arbitrary nature of these regions, and to produce algorithms which are not sensitive to this kind of switching. To simplify the discussion, we will continue to consider switched vector fields where there are only two possible vectors associated with any given input; i.e., $\sigma=\{1,2\}$. This is merely a notational convenience, however, and most of the following holds for higher multiplicity systems. The following definition reflects that we consider systems where the switching can happen arbitrarily quickly.

Definition 5 The switched vector is defined to be

$$
\begin{aligned}
& \gamma\left(q_{0}\right)=\lim _{\varepsilon \rightarrow 0} \frac{1}{\varepsilon}\left(\Phi_{\beta_{1} \varepsilon}^{g_{1}} \circ \Phi_{\beta_{2} \varepsilon}^{g_{2}} \circ \cdots \circ \Phi_{\beta_{n} \varepsilon}^{g_{2}}\left(q_{0}\right)-q_{0}\right) \\
& \text { where } \sum_{i=1}^{n} \beta_{i}=1 .
\end{aligned}
$$

This definition allows for switching to occur even as $\varepsilon \rightarrow 0$. It also avoids the complications that would otherwise arise from discontinuous functions in a derivative-based definition. Additionally, it does reduce to a classical vector when $\beta_{1}=1$, which implies that $\gamma \neq \varnothing$, the empty set. Note that $\gamma\left(q_{0}\right)$ is generally a set valued object. It is simple to show the switched vector $\gamma\left(q_{0}\right)$ of Definition 5 is the convex hull of the component vectors, i.e. $\gamma\left(g_{0}\right)=\operatorname{co}\left\{g_{i}\left(q_{0}\right) \mid i \in J\right\}$.
An interesting implication of the previously stated fact is that any switching of the above form is locally equivalent to a smooth vector field $g \in \gamma$ if we allow the switching to occur sufficiently faster than the flow is evolving. In particular, if $r$ is the number of switches that occur in time $\epsilon$, the flow associated with switching vector field is arbitrarily approximated by a smooth vector field $g$ as $r \rightarrow \infty$. For those more familiar with the formalism in [8], this is equivalent to assuming that we are only dealing with analytic selections. It should be noted that this is an extremely strong statement, particularly in view of how we use it later in Prop. 2, and represents the major weakness in the analysis carried out in this paper. Having defined a switched vector field (using the term field loosely now to allow for the set valued objects we are dealing with), we can address the issue of whether the set valued dynamics has a Lie Algebraic structure. While there is a bracket, it is not quite the most natural operator one might hope for. Nevertheless, we can now motivate the definition of a Switched Lie bracket.

Definition 6 The One Sided Uncontrolled Switched Lie bracket (IUSLB) is defined as:

$$
\begin{gathered}
{[\gamma, \eta]_{S}\left(q_{0}\right)=\lim _{\varepsilon \rightarrow 0} \frac{1}{\varepsilon^{2}}\left(\Phi_{\varepsilon}^{-g} \circ \Phi_{\varepsilon}^{-f} \circ \Phi_{\varepsilon}^{g} \circ \Phi_{\varepsilon}^{f}\left(q_{0}\right)-q_{0}\right)} \\
\forall f \in \gamma \text { and } g \in \eta \text { where } \gamma \text { and } \eta \text { are switched vectors. }
\end{gathered}
$$

Definition 6 guarantees symmetry of the flow, thereby ensuring that only second order terms arise in the limit. This also makes sense in terms of how Filippov [8] defines equations on the boundary between two sets of dynamics. Consider the following limit:

$$
\lim _{q \rightarrow N} \lim _{\varepsilon \rightarrow 0} \frac{1}{\varepsilon^{2}}\left(\Phi_{\varepsilon}^{-g} \circ \Phi_{\varepsilon}^{-f} \circ \Phi_{\varepsilon}^{g} \circ \Phi_{\varepsilon}^{f}\left(q_{0}\right)-q_{0}\right)
$$

This limit would be the multivalued map as defined on the boundary in the configuration space between two sets of dynamics (like that seen in Fig. 5). Moreover, it gives the same bracket as in Def. 6. Using this definition, one can show that $[\gamma, \eta]_{S}$ forms a convex hull of the individual Lie bracket vectors. The problem of controllability can be approached with this notion of switched vector Lie brackets. An extension of the definitions of the traditional notions of distribution and involutive distribution is needed.

\section{Definition 7 Let}

$$
\Delta_{S}=\bigcap_{\sigma \in \Sigma}\left(\operatorname{span}\left\{g_{i} \mid \quad g_{i} \in \gamma_{i} \quad i=1, \cdots, m\right\}\right)
$$

where $m$ is the number of input vectors. Similarly define the $\bar{\Delta}_{S}$ to be the span over not only the inputs, but their associated brackets.

Proposition 2 Let $r$ be the total number of switches that occur in time $T$. Then, taking the limit $r \rightarrow \infty$, the control system of Definition 2 is small time locally controllable at $q \in Q$ if $\bar{\Delta}_{S}=T_{q} \mathbb{R}^{n}$.

Proof: Here we follow the essence of the proof of Chow's theorem found in [4]. To show necessity, assume that the $k$ 
switched vectors satisfying the above property are $\gamma_{1} \cdots \gamma_{k}$. If $\bar{\Delta}_{S} \neq T Q$, then $\exists g_{i} \in \gamma_{i}$ such that $\bar{\Delta} \neq T Q$ where $\bar{\Delta}$ is the classical distribution obtained by holding the $\delta_{i}$ constant. Then, because in Def. 2 the $g_{i}$ are analytic, the classical Chow's theorem implies the system is not locally controllable.

To show sufficiency, first note that a vector $g_{i} \in \gamma_{i}$ associated with a given switching sequence is smooth in the limit as $r \rightarrow \infty$. Additionally recall that local controllability is equivalent to $\operatorname{int}\left(\mathcal{R}^{V}\left(q_{0}, \leq T\right)\right)$ being nonempty. Now we proceed by recursion. Associated with any switching sequence $\sigma$ up to time $\epsilon_{1}$ is $g_{1} \in \gamma_{1}$ where $\gamma \in \Gamma$. For $\epsilon_{1}>0$ small, $N_{1}=\left\{\phi_{t_{1}}^{\gamma_{1}} \mid 0<t_{1}<\epsilon_{1}\right\}$ is a smooth surface (of dimension 1) because $g_{1}$ is an analytic vector field.

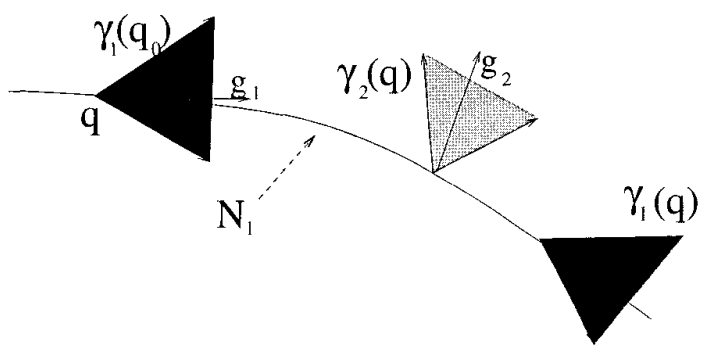

Figure 6: Proof of Controllability

Now assume that $N_{k} \in V$ is a $k$ dimensional manifold. If $k<n$, then there exists $q \in N_{k}$ and $\gamma_{k+1} \in \Gamma$ such that $\gamma_{k+1} \not \subset T_{q} N_{k}$. (i.e. $g_{k+1} \in \gamma_{k+1} \Rightarrow g_{k+1} \notin T_{q} N_{k}$ ) If this was not the case, then $\bar{\Delta}_{S} \subset T_{q} N_{k}$ for any $q$ in some open set in $N_{k}$, and the distribution restricted to that open set would be involutive. This in turn implies the existence of a switching signal $\sigma$ such that $\operatorname{dim} \bar{\Delta}_{S}=k$ which contradicts our assumption that it is equal to $n$.

Now for $\epsilon_{k+1}$ small enough, the surface

$N_{k+1}=\left\{\phi_{t_{k+1}}^{g_{k+1}} \circ \cdots \circ \phi_{t_{1}}^{g_{1}} \mid 0<t_{i}<\epsilon_{i}, i=1, \cdots, k+1\right\}$ is a $k+1$ dimensional manifold. Moreover, since $\epsilon_{i}$ can be made arbitrarily small, we can assume $N_{k+1} \subset V$. Now if $k=n, N_{k} \subset V$ is an $n$ dimensional manifold, which implies that $\operatorname{int}\left(\mathcal{R}^{V}\left(q_{0}, \leq T\right)\right)$ is nonempty.
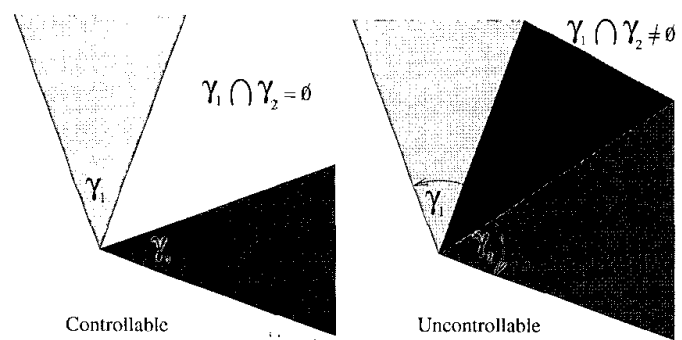

Figure 7: Controllability vs. Noncontrollability

Intuitively, Proposition 2 says that if we have some set of $n$ nominally independent control directions, there exists a switching sequence which can make two control directions the same if and only if their associated convex hulls intersect. Therefore, worst case scenario switching can make the system locally uncontrollable if and only if the convex hulls associated with the two switched vector fields intersect. One advantage of this approach is that it does lead to such a geometrically simple interpretation of the controllability condition. On the other hand, we were forced to use the assumption of taking the limit of $r \rightarrow \infty$ in order to be able to make the flow produce a smooth manifold. Moreover, this does not imply that the system is not controllable, only that it is not locally controllable. However, in the case of the rover, we are interested in local controllability so that fine maneuvering can be achieved. An equivalent statement of Proposition 2 can be found in Corollary 3, where an algebraic, as opposed to a geometric, condition for necessary and sufficient conditions on controllability is found.

Corollary 3 Let $Q, \gamma_{i}$ be as in Proposition 2. Writing $\gamma_{i}$ as $\gamma_{i}=\operatorname{co}\left\{v_{1}, \cdots, v_{l}\right\}=\sum_{i=1}^{n} \delta_{i} v_{i}$ such that $\sum_{i=1}^{n} \delta_{i}=1$ and $\delta_{i} \in[0,1]$, then the control system is locally controllable if $\operatorname{rank}\left(\bar{\Delta}\left(\gamma_{1}, \cdots, \gamma_{k}\right)=n \forall\left\{\delta_{1}, \delta_{2}, \ldots, \delta_{l_{\text {total }}}\right\} \in\right.$ $[0,1]^{l_{\text {total }}}$

For the SR7, this amounts to assuring that the determinant of a modest matrix is always nonzero, thereby reducing the question of controllability to an algebraic inequality. For the SR7, we compute the rank of the controllability matrix by computing the determinant of the algebraic condition found in Prop. 3.

$$
\operatorname{Det}\left[g_{3}, \gamma,\left[g_{3,}, \gamma\right],\left[\left[\ddot{g}_{3}, \gamma\right], \gamma\right]\right]
$$

Now, substituting in $\gamma=\delta g_{1}+(1-\delta) g_{2}$ and evaluating brackets, we get the determinant equal to $\operatorname{Cos}(\psi)$, which means that controllability is ensured so long as $\Psi \neq \frac{\pi}{2}$, where $\Psi$ is the front wheel angle.

\section{Motion Planning Primitives}

This section describes some simple motion planning techniques for overconstrained wheeled vehicles. To understand some of the complications involved with overconstrained vehicles, consider the simulation in Fig. 8 . In this simulation, the SR7 drives with constant steering wheel speed and angle. With no switching, the vehicle's center would describe a constant radius circular motion. However, in this simulation the vehicle passes over terrain regions with different friction coefficients, thereby causing switches in the dynamics. The path's non-circular geometry clearly indicates that switching can introduce considerable error. This simulation shows why standard motion planning techniques are not directly applicable. This unpredictable switching behavior may make the open loop motion plannịng problem seem insurmountable, and suggests that feedback is an appropriate strategy in this case. However, some heuristic techniques are available.

In nonholonomic systems, motion in the linearly uncontrollably directions is created by periodic inputs (or "Lie bracket motions"). This observation guided our extension of the classical Lie bracket to the switched case. With this extension, it appears true that many (though probably not 


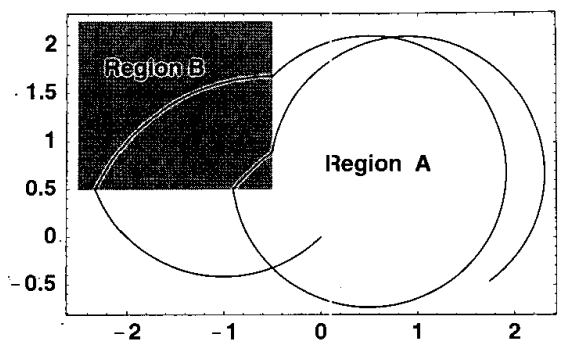

Figure 8: SR7 with Constant Wheel Angle and Speed

all) of the open loop local motion planning concepts from the classical nonholonomic literature can be adapted, using simple types of feedback, to the cverconstrained case.

In the uncontrolled switching case, the primary difficulty shifts from control of an othervise underactuated mechanism to the errors associated with switching. For motion planning purposes, one must use some form of feedback. The algorithmic approach is similar to the controlled switching case. While we wish to use the Lie bracket motion to arrive at our final state, the actual Lie bracket is $[f, \gamma]=\delta\left[f, g_{1}\right]+(1-\delta)\left[f, g_{2}\right]$. Fortunately, there is a natural choice of $\delta$. If we know something about the environment's properties, $\delta$ is chosen to be the percentage of time that the vehicle is expected to be in state $1: \delta=.5$ corresponds to equal possibility of being in one state or another. We heuristically choose to base our Lie bracket motion on the vector field $g=\delta\left[f, g_{1}\right]+(1-\delta)\left[f, g_{2}\right]$. Error is then introduced due to switching, and we iterate.

Traditionally, the use of sinusoids has arisen as the solution to optimal control problem that asks how to move a chained form system in its Lie bracket direction while minimizing $J=\int\|u\|^{2}$ [4]. We can likewise introduce an optimal control framework where the cost function is chosen to minimize a weighted cost combining power and error due to switching. To achieve this, choose

$$
J=\int \alpha\|u\|^{2}+\beta\|\varphi\|^{2}
$$

instead of $J=\int\|u\|^{2}$ as in Refs. [4]. Here $\varphi$ is the angle between $g_{1}$ and $g_{2}$ defined by the euclidian metric. I.e.,

$$
\varphi=\cos ^{-1}\left(\frac{<g_{1}, g_{2}>}{\left\|g_{1}\right\|^{2}\left\|g_{2}\right\|^{2}}\right)
$$

Equation 6 is unfortunately too complicated to yield a nice solution. However, $\varphi \approx \psi$ to third order in $q$, making

$$
J=\int \alpha\|u\|^{2}+\beta\|\psi\|^{2}
$$

a natural choice of cost function. Interestingly, this cost function is the same as in Refs. [4], except that $\|\cdot\|$ is now associated with a new metric. This choice of cost function encapsulates the fact that the more one is willing to keep the wheels pointing close to straight forward (and therefore is willing to move increasingly slowly in the Lie bracket direction) the more one can reduce error. In the simulation in Fig. 9, this method converges in only two iterations to the goal state of $\left[4,4, \frac{\pi}{4}\right]^{T}$.

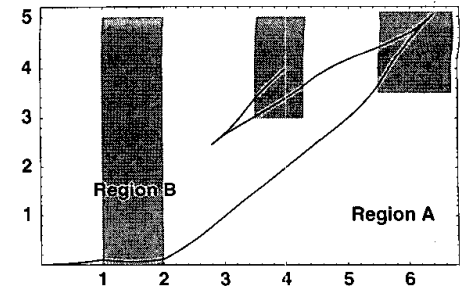

Figure 9: $\mathrm{X}$ and $\mathrm{Y}$ Coordinates for SR7

\section{Conclusions}

Standard nonholonomic motion planning and control theories can not be directly applied to the potentially important class of overconstrained wheeled vehicles. This paper outlined some initial steps towards a motion planning and control theory for such vehicles. We extended Chow's theorem to both the controlled and uncontrolled switching cases. While we demonstrated useful primitives for open-loop motion planning, a formal and general motion planning scheme is still forthcoming. The uncertainty associated with the uncontrolled switching case clearly suggests the investigation of feedback schemes to stabilize such systems. Finally, we expect that further investigation of the likely strong relationship between the simplex method in LP theory and the numerical techniques required to solve for the minimum of the dissipation functional will be fruitful.

Acknowledgements: This work was partially supported by the National Science Foundation (Grant \#NSF9402726) through their Engineering Research Center (ERC) program.

\section{References}

[1] A.M. Bloch, P. S. Krishnaprasad, J.E. Marsden, and R.M. Murray. Nonholonomic mechanical systems with symmetry. Arch. Rational Mech. Anal., 136(1):21-99, 1996.

[2] A.R. Teel, R.M. Murray, and G. Walsh. Nonholonomic control systems: From steering to stabilization with sinusoids. Int. J. of Control, 62(4):849-870, 1995.

[3] J.C. Alexander and J.H. Maddocks. On the kinematics of wheeled vehicles. The International Journal of Robotics Research, 8(5):15-27, October 1989

[4] S. Sastry. Nonlinear Systems: Analysis, Stability, and Control. Springer, 1999.

[5] B. Goodwine and J. W. Burdick. Controllability of kinematic control systems on stratified configuration spaces. (to appear) IEEE Trans. on Automatic Control, 2000.

[6] T. Murphey and J. W. Burdick. Issues in controllability and motion planning for overconstrained wheeled vehicles. In Proc Int Conf Math Theory Networks Systems (MTNS), Perpignan, France, 2000.

[7] D. Liberzon and A. S. Morse. Benchmark problems in stability and design of switched systems. PrePrint, 1999.

[8] A.F. Filippov. Differential Equations with Discontinuous Right Hand Sides. Kluwer Academic Publishers, 1988. 